\title{
Analysis and Evaluation of Learning/Training Time Convergence Associated with E-learners Using Artificial Neural Networks Modeling
}

\author{
Hassan M. H. Mustafa ${ }^{1}$, Fadhel Ben Tourkia ${ }^{2}$ \\ ${ }^{1,2}$ Computer Engineering Department, Al-Baha Private College of Sciences, Al-Baha, Kingdom of Saudi Arabia.
}

\begin{abstract}
The objective of this piece of research is to interpret and investigate systematically an observed brain functional phenomenon which associated with proceeding of e-learning processes. More specifically, this work addresses an interesting and challenging educational issue concerned with dynamical evaluation of e-learning performance considering convergence (response) time. That's based on an interdisciplinary recent approach named as Artificial Neural Networks $\left(A N N^{\underline{s}}\right)$ modeling. Which incorporate Nero-physiology, educational psychology, cognitive, and learning sciences. Herein, adopted application of neural modeling results in realistic dynamical measurements of e-learners' response time performance parameter. Initially, it considers time evolution of learners' experienced acquired intelligence level during proceeding of learning / training process. In the context of neurobiological details, the state of synaptic connectivity pattern (weight vector) inside e-lear0.3ner's brain-at any time instant-supposed to be presented as timely varying dependent parameter. The varying modified synaptic state expected to lead to obtain stored experience spontaneously as learner's output (answer). Obviously, obtained responsive learner's output is a resulting action to any arbitrary external input stimulus (question). So, as the initial brain state of synaptic connectivity pattern (vector) considered as pre-intelligence level measured parameter. Actually, obtained e-learner's answer is compatibly consistent with modified state of internal / stored experienced level of intelligence. In other words, dynamical changes of brain synaptic pattern (weight vector) modify adaptively convergence time of learning processes, so as to reach desired answer.

Additionally, introduced research work is motivated by some obtained results for performance evaluation of some neural system models concerned with convergence time of learning process. Moreover, this paper considers interpretation of interrelations among some other interesting results obtained by a set of previously published educational models. The interpretational evaluation and analysis for introduced models results in some applicable studies at educational field as well as medically promising treatment of learning disabilities. Finally, an interesting qualitative comparative study has been briefly reviewed by the end of this paper. It considered the comparative analogy between performance evaluation of behavioral ANNs modeling; versus smart optimization in Ant Colony System (ACS).
\end{abstract}

Keywords: Artificial Neural Network Modeling, E-Learning Performance Evaluation, Synaptic Connectivity, Ant Colony System.

\section{Introduction}

The last decade of previous century(1990-2000)named as the Decade of the brain, that after referring to WHITE HOUSE OSTP REPORT(U.S.A.) which declared in 1989[1]. Consequently, educationalists as well as computer engineering scientists have adopted research approach associated with natural intelligence (recent computer generation), and basic brain functions (learning and memory). Additionally, this approach has been tightly related to developed trends in information technology to attain systematic analysis and performance evaluation of various learning processes. It is worthy to note that recent evolutionary interdisciplinary trends have been adopted by educationalists incorporating Nero-physiology, psychology, and cognitive learning sciences. Herein, specifically this paper motivated by the work of Grossberg in 1988, therein the concept of natural intelligence introduced [2]. Consequently, artificial neural networks ( $\mathrm{ANN}^{\mathrm{s}}$ ) modeling has been adopted 
to investigate systematically mysteries of the most complex biological neural system(human brain). Accordingly, evolutionary interdisciplinary trends have been adopted by educationalists, neurobiologists, psychologists, as well as computer engineering researchers in order to carry out realistic investigations for some critical challenging educational issues [3][4][5][6][7][8]. Due to currently rapid development in the research field of learning sciences which represented by a growing community internationally. Many experts have been recently recognized there interest in facing educational systems' challenging phenomenal issues. That have been given via current advances in communication, and information technology-mediated learning. Moreover, a set of very recently approach papers have been published having a special attention towards three interdisciplinary educational issues. Namely, noisy learning environment, overcrowded classrooms, and not well qualified instructors[9][10][11].

Generally, evaluation of learning performance is a challenging, interesting, and critical educational issues [12][13][14][15][16]. Specifically, considering academic performance measurement of e-learning systems some interesting papers have been published as introduced at [17][18][19][20][21]. Educationalists have been in need to know how neurons synapses inside the brain are interconnected together, and communication among brain regions[8]. By this information they can fully understand how the brain's structure gives rise to perception, learning, and behavior, and consequently, they can investigate well the learning process phenomenon[19][20]. This paper presents an investigational approach getting insight with e-learning evaluation issue adopting (ANN ${ }^{\mathrm{s}}$ ) modeling. The suggested model motivated by synaptic connectivity dynamics of neuronal pattern(s) inside brain which equivalently called as synaptic plasticity while coincidence detection learning (Hebbian rule) is considered [22]. The presented interdisciplinary work aims to simulate appropriately performance evaluation issue in e-learning systems with special attention to face to face tutoring [23] .That purpose fulfilled by adopting learner's convergence (response) time, (as an appropriate metric parameter) to evaluate his interaction with elearning course material(s). In fact this metric learning parameter is one of learning parameters recommended for using in educational field by most of educationalists. In practice, it is measured by a learner's elapsed time till accomplishment of a pre-assigned achievement level (learning output) [21] [23][14]. Thus, superior quality of evaluated e-leaning system performance could be reached via global decrement of learners' response time. Accordingly, that response time needed-to accomplish pre-assigned learners' achievement- is a relevant indicator towards quality of any-under evaluation- learning system. Obviously, after successful timely updating of dynamical state vector (inside e-learner's brain) pre-assigned achievement is accomplished[12]. Consequently, assigned learning output level is accomplished if and only if connectivity pattern dynamics (inside learner's brain) reaches a stable convergence state,(following Hebbian learning rule). In other words, connectivity vector pattern associated to biological neuronal network performs coincidence detection to input stimulating vector. i.e. inside a learner's brain, dynamical changes of synaptic connectivity pattern (weight vector) modifies adaptively convergence time, so as to deliver (output desired answer). Hence, synaptic weight vector has become capable to spontaneous responding (delivering correctly coincident answer) to its environmental input vector (question)[12][13][15][24][25]. Interestingly, some innovative research work considered systematically the observed analogy between learning process concerned with smart swarm intelligence (Ant Colony Systems).Versus learning performance at behavioral neural systems have been published at [26] [27][28][29][30][31][32][33].

The rest of this paper is organized as follows. The next second section a review for performance evaluation techniques is presented. Selectivity criteria used in ANN models are briefly reviewed at the third section. At the fourth section, modeling for three learning phases face to face (learning under supervision), unsupervised learning (self-study), and learning by interaction with other followers (e-learners) is presented. Experimental measurement of response time and simulation results are shown at the fifth section. This section considers the effect of gain factor of ANN on the time response in addition to comparative analogy between the gain factor effect-during learning process evaluation- in neural networks systems. Versus the impact of intercommunication cooperative learning cooperative learning parameter-while solving Traveling Sale Man(TSP)Problem- in Ant Colony System (ACS). At the sixth section, some conclusions and suggestions for future work are presented. Finally, all of cited references are given at the last seventh section. 


\section{Performance Evaluation Techniques}

The most widely applicable techniques considering performance evaluation of complex computer systems are presented along with analysis of statistical modeling, and simulation for some experimental results measurement (given in the fifth section). More recently, self assessment for Blended Learning Performance has been published,[20]. Herein, all of three techniques are presented, with giving special attention to simulation using ANN modeling for learners' brain functions. Quantitative evaluation of timely updating brain function is critical for the delivery of a pre-assigned learning output level for a successful e-learning system. More precisely, inside a learner's brain, dynamical changes of synaptic connectivity pattern(weight vector)modifies adaptively convergence time, so as to deliver (output desired answer) [19][25].

\subsection{Selecting an Appropriate Learning Parameter}

Referring to some educational literature one of the evaluating parameter for learning processes is learning convergence time (equivalently called as response time) [19].By more details, at e-educational field practice while a learning processes proceeds, e-learners are affected naturally by technical characterizations as well as technological specifications of the interactive learning environment. Thus, learners have to submit their desired achievements (output learning levels) finally, by successive timely updated interaction with learning environmental conditions. This is well in agreement to the unsupervised (autonomous) learning paradigm following Hebbian rule [3][22]. in case of self-study learning. Conversely, considering the case of second learning way concerned with supervised learning (with a teacher) paradigm. It would be relevant to follow error correction learning algorithm as an ANN model [16][24]. Accordingly, two ANN models (supervised and unsupervised), are suggested for realistic simulation of both face to face learning ways :from tutor and from selfstudy, respectively [23].

Accordingly, learners' updated performance is directly (globally and/or individually) influenced by communication engineering efficiency of interactive channels. Such channels are practically non-ideal and contaminated by various types of noise sources accounting to some impairment to acquired learning data [23]. Of course, learning impairment accounts to worst learning performance with lower learning rate [23] [26]. Interestingly: poverty as a social phenomenon considered as environmental noise affecting learning reading (comprehension) performance. This considered evidence for analogy between poverty and noise is strongly supported by recently announced findings at [2] .That supporting is given by wording : "Lower income kids performed at a lower level than others high-income children in reading comprehension and mathematical calculations but were competitive in basic cognition, memory and reading skills, indicating that poverty may affect development at the level where different abilities must be combined, such as verbal skills and memory, in the case of reading comprehension". Moreover, the issue concerned with relation between noise effect and learning quality (measured by learning rate value) is discussed in details at a recent research work [21].

Obviously, various human learning original phenomena such as Psychological, Cognitive styles, ......etc. , should have significant influence on learning environmental performance[ ]. Conclusively, in practice; learning processes are virtually and/or actually vulnerable to non-ideal noisy data due to environmental conditions. Moreover, it is noticed learners' obtained achievements depends upon effectiveness of face to face tutoring process. Additionally, successful learners' interaction must emerge from the software quality of applied learning module packages. So, any assigned learning output level is accomplished if and only if connectivity pattern dynamics (inside learner's brain) reaches a stable convergence state. At section 6, a review for noise effect on learning performance is presented. Moreover, initial state of synaptic connectivity vector has effect on convergence time of learning processes [23][26]. Accordingly, response time needed to accomplish pre-assigned learners' achievement is a relevant indicator towards quality of any under evaluation system. Obviously, after successful timely updating of dynamical state vector (inside e-learner's brain) pre-assigned achievement is accomplished [21][25]. Consequently, assigned learning output level is accomplished if and only if connectivity pattern dynamics (inside learner's brain) reaches a stable convergence state (following Hebbian learning rule). In other words, connectivity vector pattern associated to biological neuronal network performs coincidence detection to input stimulating vector. i.e. inside a learner's brain, dynamical changes of synaptic connectivity pattern (weight vector) modifies adaptively convergence time, so as to deliver (output desired answer) [25] . 


\subsection{Examinations in E-Learning Systems}

Neural networks' modeling has been adopted in fulfillment of better learning achievements during face to face tutoring. Accordingly, quantitative analysis of e-learning adaptability performed herein, via assessment of matching between learning style preferences and the instructor's teaching style and/or e-courses material.[27].More specifically, e-learning system performance evaluation, time response parameter applied to measure any of e-learners' achievement. Thus e-learner has to subject to some timely measuring examination that is composed as Multi choice questions. Hence, this adopted examination discipline is obviously dependent upon learners' capability in performing selectivity of correct answer to questions they received [21]. Consequently, to accomplish a pre-assigned achievement level, stored experience inside learner's brain should be able to develop correct answer up to desired(assigned)level. In the context of biological science selectivity function proceeds (during examination time period) to get on either correct or wrong answer to received questions spontaneously. Accordingly, the argument of selectivity function is considered virtually as the synaptic pattern vector(inside brain)is modified to post training status. Hence, selected answer results in synaptic weight vector has become capable to respond spontaneously(delivering correctly coincident answer) to its environmental input vector (question) [12][13].

\section{Selectivity Criteria[34]}

Referring to adopted performance evaluation technique of e-learning systems by response time parameter. Accomplishment of a learner's output is dependent on the optimum selection of correct answer as quick as possible. So it is well relevant to present ANN models that capable to perform selectivity function while solving some critical problems. Consequently, the goal of this section is that to give -in brief- an overview over mathematical formulations of selectivity criteria adopted by various neural network models. This overview sheds light on the selectivity criterion adopted by our proposed model. Presented selectivity criteria are given in a simplified manner for four neural network models adopting adaptive selectivity criterion, as follows:

\subsection{Selectivity Criterion by Grandmother Models [35]}

On the basis of grandmother modelling, a simple sorting system has been constructed using a set of grandmother cells. That implies, each neuron has been trained in order to respond exactly to one particular input pattern. In other words, each neuron has become able (after training) to recognize its own grandmother. Appling such models in real world, they have been characterized by two features. Firstly, a lot number of grandmother cells are required to implement such grandmother model. That is due to the fact each cell is dedicated to recognize only one pattern. Secondly, it is needed to train that simple sorting network possible grandmother pattern to obtain correct output response. Consequently, all synaptic weight values at this model have to be held up unchanged (fixed weights). Hence, it is inevitably required to either add new grandmother cell(s), to recognise additional new patterns or, to modify weights of one or more existing cells to recognise that new patterns.

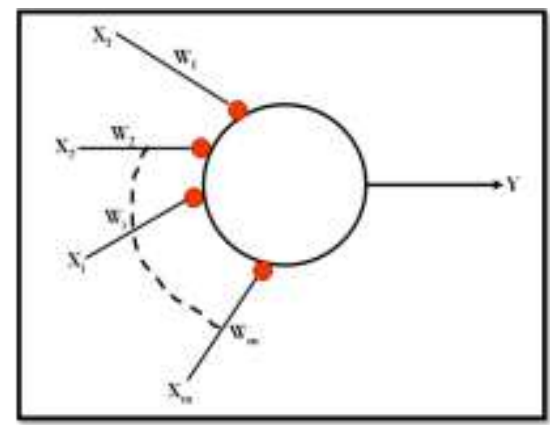

Fig. 1: Illustrates a single grandmother cell (artificial neuronal cell) that works as processing element. (Adapted from [35])

The above grandmother model could be described well by following mathematical formulation approach. The output of any grandmother cell (neuron) is a quantizing function defined as follows:

$\phi(a)=\left\{\begin{array}{lll}0 & \text { when } & a<0 \\ 1 & \text { when } & a \geq 1\end{array}\right.$ 
Then the output y is represented by

$y=\phi(U-\Theta)$

Where $\mathrm{U}$ is defined as

$U=\sum_{i=1}^{m} w_{i} x_{i}$

That model utilizes a set of grandmother neuronal cells (nodes). Each of these nodes responds exactly to only one particular input data vector pattern. Therefore, for some specific input vector pattern with $\mathrm{m}$ dimension is needed to make only one of model nodes to fire selectively to it.

\section{2. Kohonen's Selectivity Criterion[36][37[38]}

The most famous approach of neuronal modelling based on selectivity is proposed by T.Kohonen and applied for Self Organizing Map (SOM), [38]. The SOM is based on vector input data to Kohonen neuronal model. That input is a vector data pattern developed as to change the status of the model. The changes are based on incremental stepwise correction process. The original algorithm of SOM aims to determine what so called winner take all (WTA) function. That function is referred to some physiological selectivity criterion applied as to define initially the function $\mathrm{C}$ that to search for $\mathrm{m}_{\mathrm{i}}(\mathrm{t})$ to be closest to $\mathrm{x}(\mathrm{t})$.

$C=\arg \min _{i}\left\{\left\|x(t)-m_{i}(t)\right\|\right\}$

where $\mathrm{x}(\mathrm{t})$ is an $\mathrm{n}$-dimensional vector data as one input sample, and $\mathrm{m}_{\mathrm{i}}(\mathrm{t})$ is a spatially ordered set of vector models arranged as a grid, and $t$ is a running index of input samples and also index of iteration steps. The iterative process supposed to be continuous by time $(t \rightarrow \infty)$ as to obtain the asymptotic values of the $\mathrm{m}_{\mathrm{i}}$ constitute the desired ordered projection at the grid.

\subsection{Hopfield Network Selectivity Model [24]}

It is proved to be closely attached with that network computational power. To obtain decisions in some optimisation problems, the computational power is demonstrated by the Hopfield NN model selectivity. That, it means the ability to select one of possible answers that model might give. Therein, resulting selectivity pattern (for all possible solutions) shown in a form of histogram. As numerical example, the value of selectivity of Hopfield neural network model was about $10^{-4}-10^{-5}$. That when it is applied to solve travelling salesman problem (TSP) considering 100 neurons. That given value of selectivity is the fraction of all possible solutions. In practice, it is noticeable that by increasing number of neurons comprising Hopfield network, selectivity of the network expected to be better (increased). The cost function concept supports the above presented selectivity criterion in TSP. By referring to Eq. (1) given at subsection (3.1), pattern of vector pairs are $\bar{x}$ and $\bar{y}$ the model are respectively called key and stored patterns of vectors'. The concept of cost function is adopted as to measure of how far away we are from optimal solution of memorization problem. Proposed mathematically, illustrations of cost is a function of observations and the problem becomes that to find the model $f$ which minimises $\mathrm{C}$ value $C=E\left\lfloor f(x)-\left.Y\right|^{2}\right\rfloor$

when we have only $\mathrm{N}$ samples of vector pairs drawn from distribution.

$\hat{C}=\frac{1}{N} \Sigma\left|f\left(x_{i}\right)-Y_{i}\right|^{2}$

\subsection{Selectivity Criterion for Learning by Interaction with Environment [34]}

It is worthy to note that selectivity condition considers a network model adopting artificial neurons with threshold (step) activation function as shown in the above at Fig.1. The necessary and sufficient condition for some neuron to fire selectively to a particular input data vector (pattern) is formulated mathematically as given in below. Consider the particular input pattern vector given by: $\mathrm{x}_{\mathrm{c}}$

$x_{c}=\left(x_{c 1}, x_{c 2}, \ldots x_{c n}\right)$ 


\section{Hence,}

$$
\begin{aligned}
& \Theta<U_{c} \\
& \Theta \geq U_{c}+W_{m}
\end{aligned}
$$

Where $\Theta$ is the fixed threshold value controlling firing of the neuron.

\section{for any m satisfying}

$$
\begin{aligned}
& \mathrm{O}=x_{c m} \\
& \mathrm{O} \geq U_{c}-W_{m}
\end{aligned}
$$

for any m satisfying

$$
\begin{aligned}
& 1=x_{c m} \\
& U_{c}=\sum_{i=1}^{n} W_{i} x_{c i}
\end{aligned}
$$

\section{Modeling of E-Learning Performance}

The figure in below illustrates the interrelations among the components of e-learning process presenting the face to face tutoring between the instructor and e-learner.

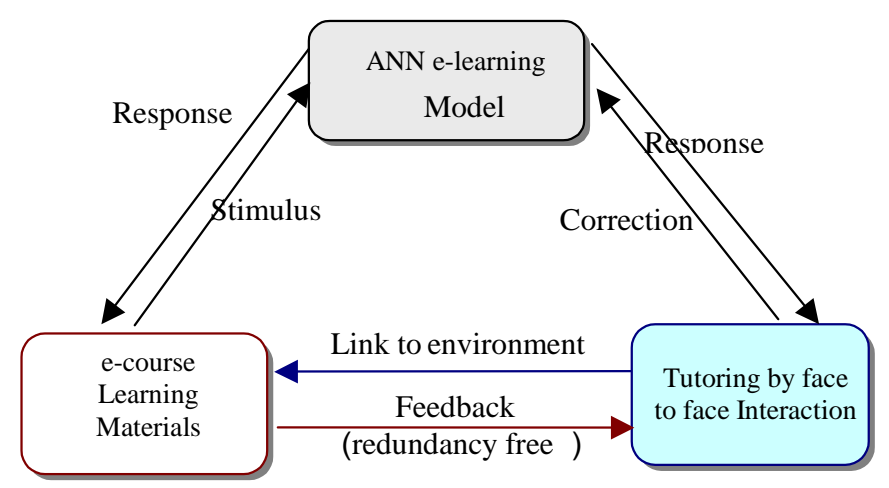

Fig 2. A general view for interactive educational process presenting face to face Interaction.

\subsection{Modeling of Face to Face Tutoring [23]}

In face to face tutoring, the phase of interactive cooperative learning is an essential paradigm aiming to improve any of e-Learning Systems' performance. In more details, face to face tutoring proceeds with three phases (Learning from tutor, Learning from self-study, and Learning from interaction with fellow learners). it has been declared that cooperative interactive learning among e-learning followers (studying agents learners).That phase contributes about one fourth of e-learning academic achievement (output) attained during face to face tutoring sessions [23]. At this subsection both of two phases concerned with the first and second phases are molded by one block diagram (Figure 3). However two diversified mathematical equations are describing the two phases separately. At the next subsection cooperative learning is briefly presented by referring to Ant Colony System (ACS) Optimization. 


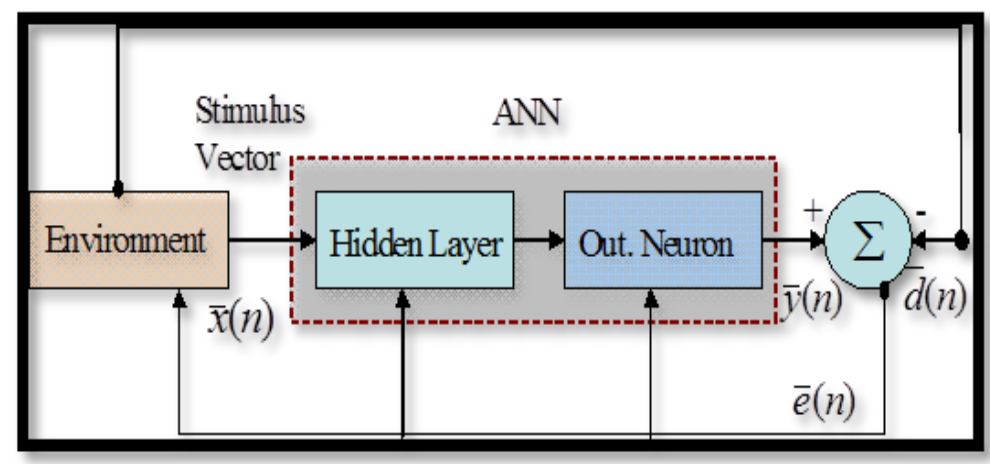

Fig.3: Block diagram for learning paradigm adopted for quantifying creativity adapted from [24].

The error vector at any time instant (n) observed during learning processes is given by:

$\bar{e}(n)=\bar{y}(n)-\bar{d}(n)$

Where

$\bar{e}(n)$ : Error correcting signal controlling adaptively

$\bar{y}(n)$ : The output signal of the model

$\bar{d}(n)$ : Numeric value(s) of the desired /objective parameter of learning process (generally as a vector).

Referring to above figure 5; following equations are considered:

$\mathrm{V}_{\mathrm{k}}(\mathrm{n})=\mathrm{X}_{\mathrm{j}}(\mathrm{n}) \mathrm{W}^{\mathrm{T}}{ }_{\mathrm{kj}}(\mathrm{n})$

$\mathrm{y}_{\mathrm{k}}(\mathrm{n})=\varphi\left(\mathrm{V}_{\mathrm{k}}(\mathrm{n})\right)=1 /\left(1+\mathrm{e}_{\mathrm{k}}^{-\lambda v}(\mathrm{n})\right.$

$\mathrm{e}_{\mathrm{k}}(\mathrm{n})=\left|\mathrm{d}_{\mathrm{k}}(\mathrm{n})-\mathrm{y}_{\mathrm{k}}(\mathrm{n})\right|$

$\mathrm{W}_{\mathrm{kj}}(\mathrm{n}+1)=\mathrm{W}_{\mathrm{kj}}(\mathrm{n})+\Delta \mathrm{W}_{\mathrm{kj}}(\mathrm{n})$

Where: $\mathrm{X}$ input vector, $\mathrm{W}$ weight vector, $\varphi$ is the activation function, $\mathrm{y}$ is the output, $\mathrm{e}_{\mathrm{k}}$ the error value, and $\mathrm{d}_{\mathrm{k}}$ is the desired output. Noting that $\Delta \mathrm{W}_{\mathrm{kj}}(\mathrm{n})$ the dynamical change of weight vector value.

The above four equations are commonly applied for both learning phases, supervised (Learning from tutor), and unsupervised (Learning from self-study). The dynamical change of weight vector value specifically for supervised phase is given by equation:

$\Delta \mathrm{W}_{\mathrm{kj}}(\mathrm{n})=\eta \mathrm{e}_{\mathrm{k}}(\mathrm{n}) \mathrm{x}_{\mathrm{j}}(\mathrm{n})$

Where $\eta$ is the learning rate value during learning process for both learning phases. However, for unsupervised paradigm, dynamical change of weight vector value is given by equation:

$\Delta \mathrm{W}_{\mathrm{kj}}(\mathrm{n})=\eta \mathrm{y}_{\mathrm{k}}(\mathrm{n}) \mathrm{x}_{\mathrm{j}}(\mathrm{n})$

\subsection{Gain Factor versus Learning Convergence}

Referring to [2][17][40], learning by coincidence detection is considered. Therein, angle between training weight vector and an input vector have to be detected. Referring to [40], the results of output learning processes considering Hebbian rule are following the equation:

$y=\left(1-e^{-\lambda t}\right)$

The above equation performs analogously to gain factor (slope) in classical sigmoid function [2].

$y(t)=\frac{1}{1+e^{-\lambda t}}$

However, equation (8) performs versus time closely similar to odd sigmoid function given as

$y(t)=\frac{1-e^{-\lambda t}}{1+e^{-\lambda t}}$

For

$0 \leq t \leq \infty$ 


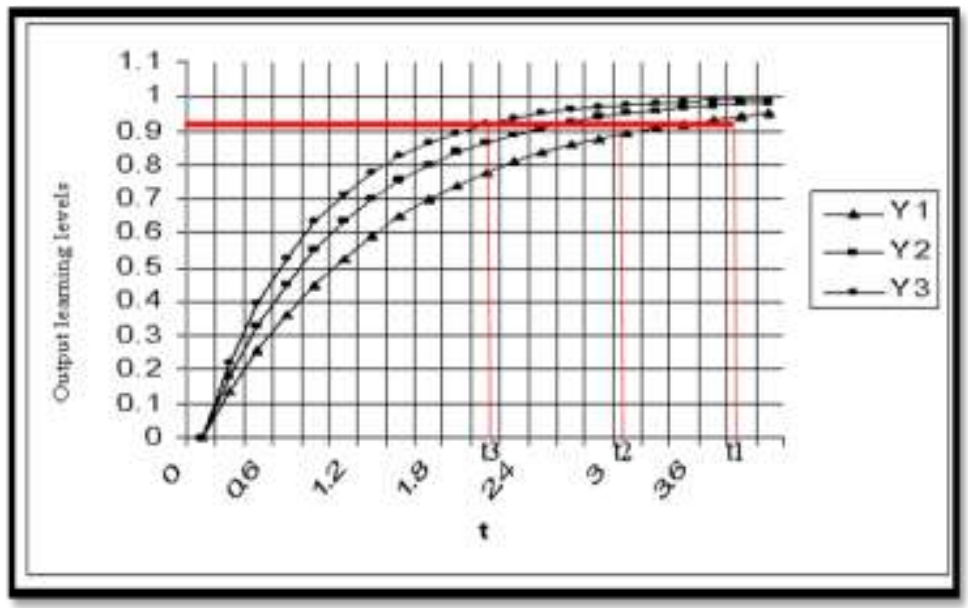

Fig.4: Illustrates three different learning performance curves $Y_{1} \& Y_{2}$ and $Y_{3}$ that converge at time $t_{1} \& t_{2}$, and $t_{3}$ considering different gain factor values $\lambda_{1} \& \lambda_{2}$, and $\lambda_{3}$.(adapted from [40]).

Referring to the above Fig.4; the three curves shown represent different individual levels of learning. Curve $\left(\mathrm{Y}_{2}\right)$ is the equalized representation of both forgetting and learning factors[40]. However curve $\left(\mathrm{Y}_{1}\right)$ shown the low level of learning rate (learning disability) that indicates the state of angle between synaptic weight vector and an input vector . Conversely, the curve $\left(\mathrm{Y}_{3}\right)$ indicates better learning performance that exceeds the normal level of learning at curve $\left(\mathrm{Y}_{2}\right)$. Consequently learning time convergence decreases as shown at Fig.6, $\left(\mathrm{t}_{1} \& \mathrm{t}_{2}\right.$, and $\left.\mathrm{t}_{3}\right)$ three different levels of learning performance curves representing: normal, low, and better cases shown at curves $\mathrm{Y}_{1} \& \mathrm{Y}_{2}$ and $\mathrm{Y}_{3}$ respectively.

\section{Simulation Results}

\subsection{Gain factor values $(\lambda)$ versus response time}

The graphical simulation results illustrated in the below Fig. 5, gain factor effect on improving the value of time response measured after learning process convergence, [41]. These four graphs at Fig.7 are concerned with the improvement of the learning parameter response time (number of training cycles). That improvement observed by increasing of gain factor values $\lambda(0.5,1,10$, and 20$)$ that corresponds to decreasing respectively number of training cycles by values (10,7.7,5,and3) cycles, (on approximate averages).

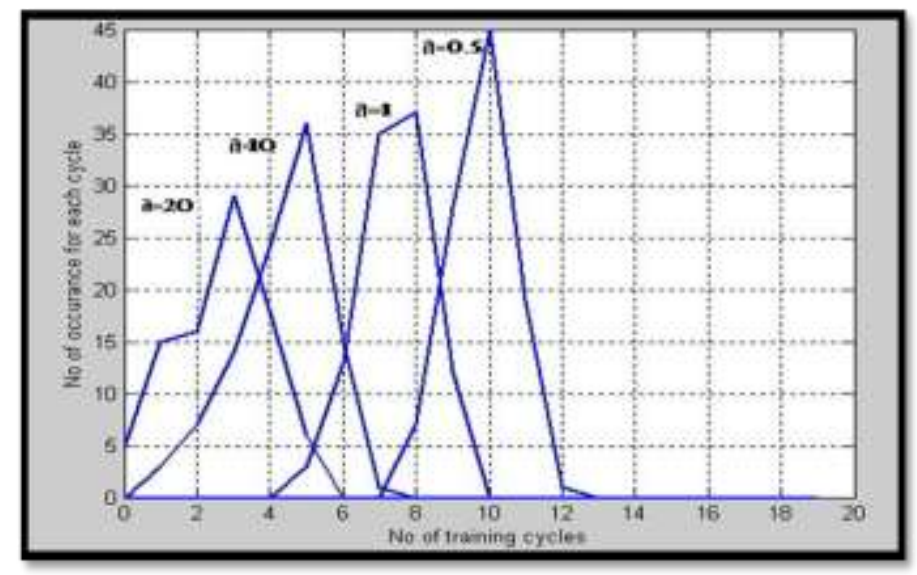

Fig.5:. Illustrates improvement of average response time (no. of training cycles) by increase of the gain factor values.

\subsection{Effect of Neurons' Number on Time Response[19][41]}

The following simulation results show how the number of neurons may affect the time response performance. Those graphical presented results show that by changing number of neural cells $(14,11,7,5$, and 3 ); during interaction of students with e-learning environment, the performance observed to be improved by increase of 
number of neuronal cells (neurons). That is shown at figures: $(8,9,10,11,12)$ respectively; for fixed learning rate $=0.1$ and gain factor $\lambda=0.5$.

No. of occurrences for each Time

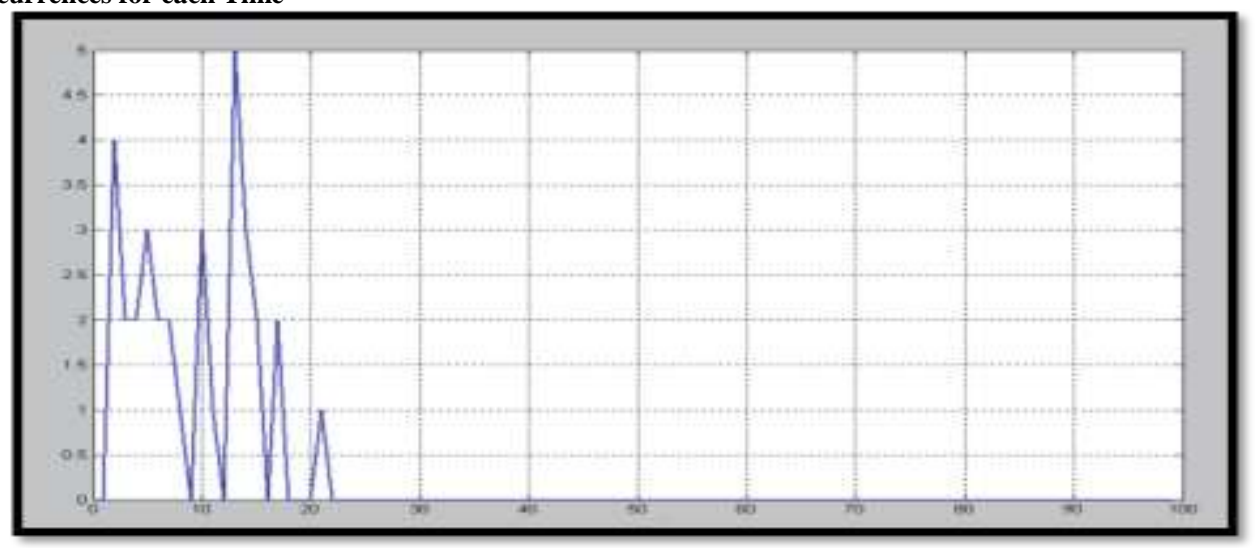

Time (No. of training cycles)

Fig: 6 Considering \# neurons= 14

No. of occurrences for each Time

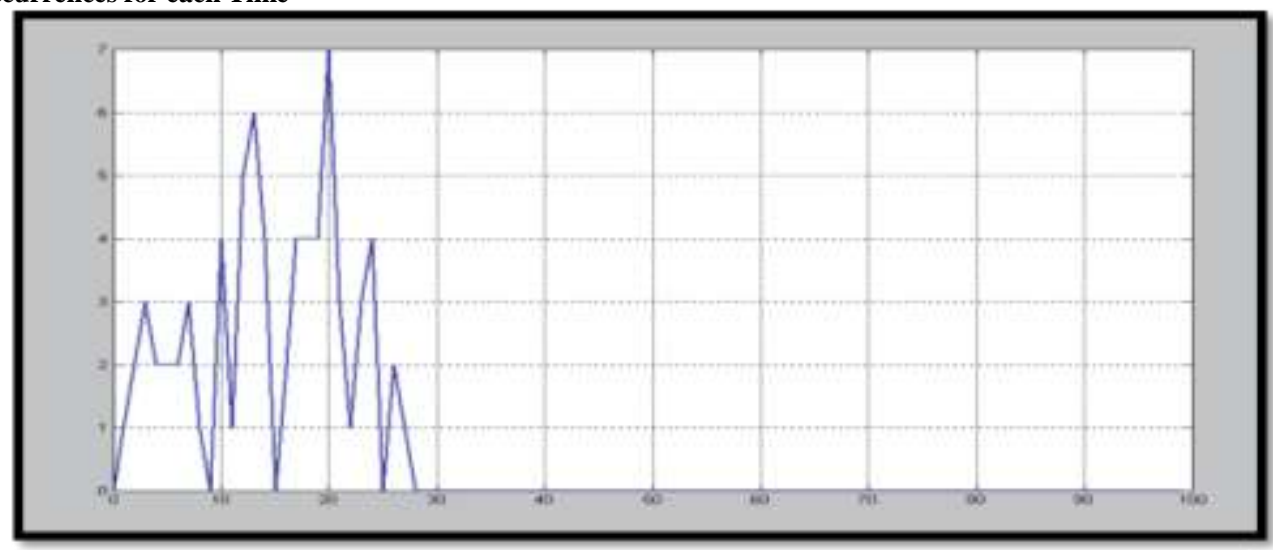

Time (No. of training cycles)

Fig.: 7 Considering \# neurons= 11

No. of occurrences for each Time

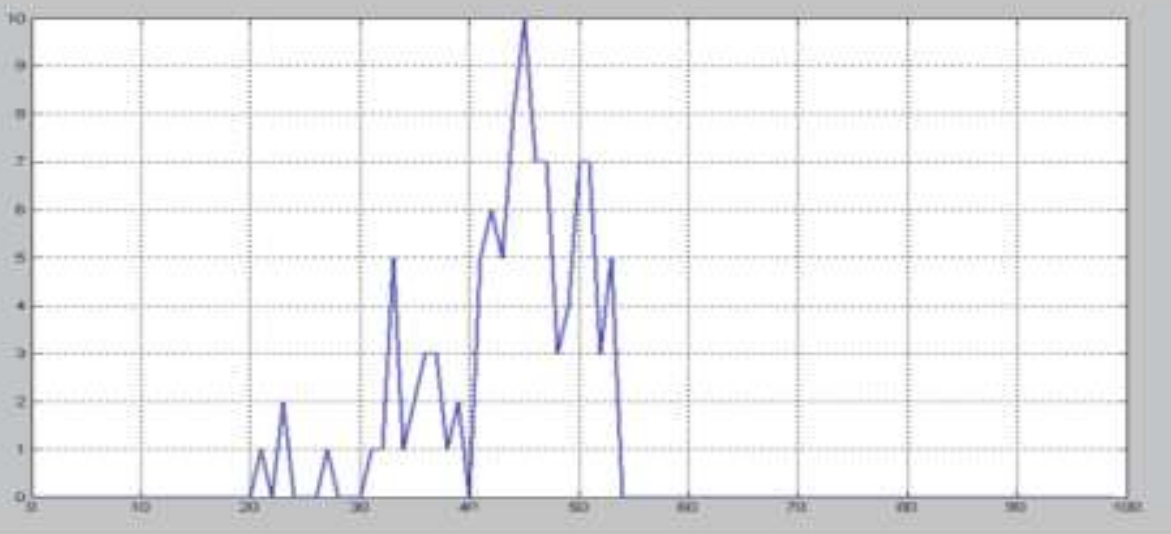

Time (No. of training cycles)

Fig.: 8 Considering \# neurons= 7

No. of occurrences for each Time 


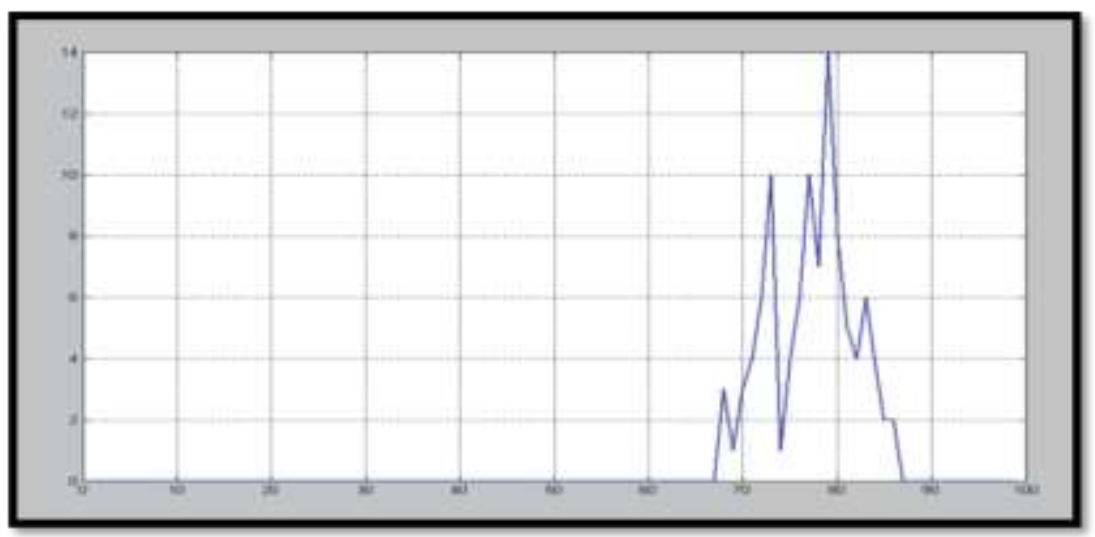

Time (No. of training cycles)

Fig. 9 : Considering \# neurons= 5

No. of occurrences for each Time

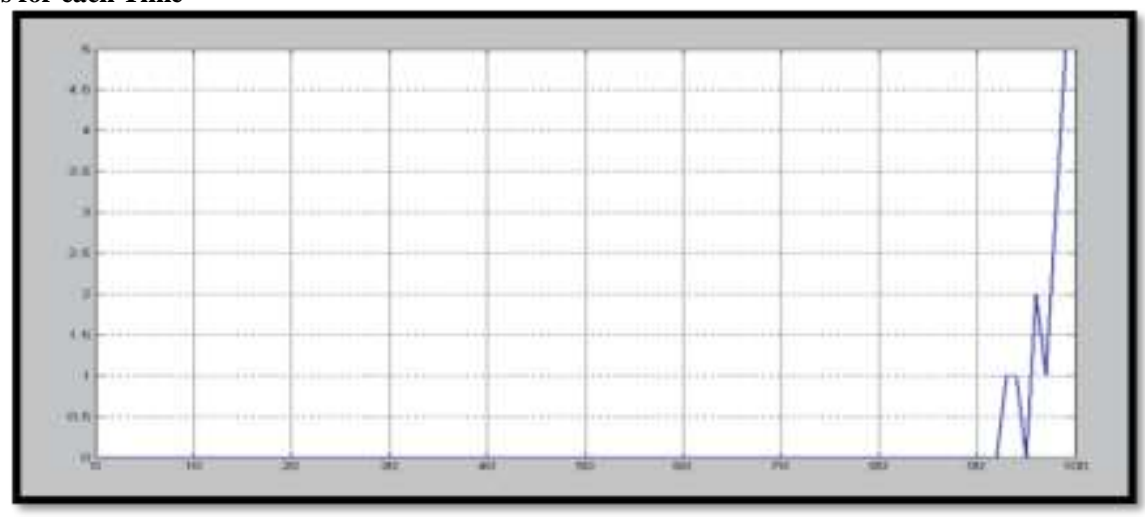

Time (No. of training cycles)

Fig. 10 : Considering \# neurons= 3

Referring to Figure 11 , It is noticed that statistical learning rate variations (on the average values) are related versus corresponding selectivity convergence (response) time. That measured convergence(response) time is presented considering the number of iteration cycles. Obtained output results corresponding to learning rate values $(\eta)(0.1,0.2,0.4,0.6$, and 0.8$)$, are given, as(330, 170,120, 80, and 40)iteration training cycles respectively . Consequently, convergence time (number of training cycles) is inversely proportional to the corresponding learning rate values. Moreover, it is an interesting remark that under more noisy environmental conditions, learning rate tends to have lower value. Conversely, e-learners performed learning rate improvement by interaction with environment, implies increase of their stored experience intrinsically via there synaptic connectivity patterns . Conclusively, such e-learners have become capable of responding spontaneously to input environmental stimuli(Questions) in an optimal manner (Desired answer) [2][19].

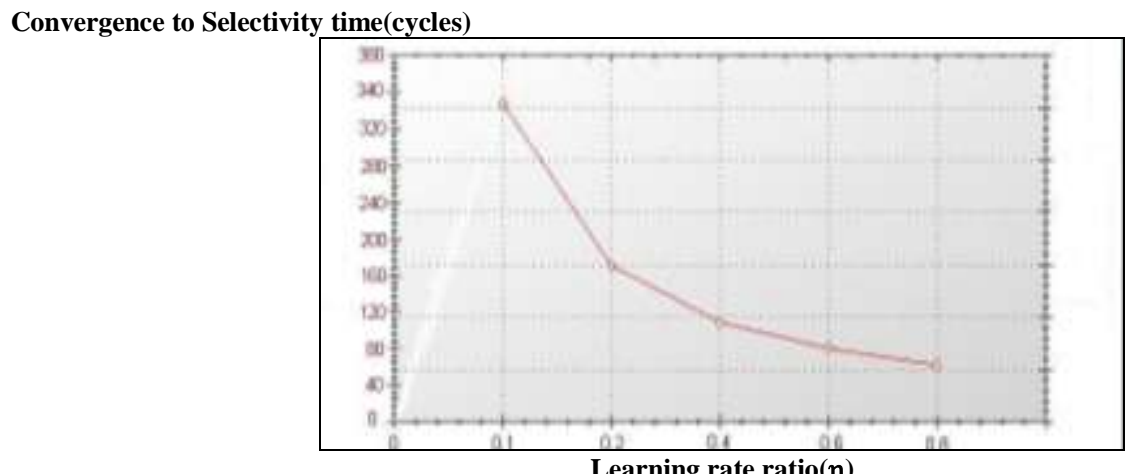

Fig.11 : Illustrates the average (of statistical distribution) for selectivity response time (number of iteration cycles) versus different learning rate ratio values $(\eta)$ 


\subsection{Analogy of Behavioral learning versus Cooperative Learning By ACS [41][42][43]}

Referring to Fig. 12 given in below, ants are moving on a straight line that connects a food source to their nest. It is well known that the primary means for ants to form and maintain the line is a pheromone trail. Ants deposit a certain amount of pheromone while walking, and each ant probabilistically prefers to follow a direction rich in pheromone(Fig.12 A). This elementary behaviour of real ants can be used to explain how they can find the shortest path that reconnects a broken line after the sudden appearance of an unexpected obstacle has interrupted the initial path (Fig.12 B). In fact, once the obstacle has appeared, those ants which are just in front of the obstacle cannot continue to follow the pheromone trail and therefore they have to choose between turning right or left. In this situation we can expect half the ants to choose to turn right and the other half to turn left. A very similar situation can be found on the other side of the obstacle (Fig.12 C). It is interesting to note that those ants which choose, by chance, the shorter path around the obstacle will more rapidly reconstitute the interrupted pheromone trail compared to those which choose the longer path. Thus, the shorter path will receive a greater amount of pheromone per time unit and in turn a larger number of ants will choose the shorter path. Due to this positive feedback (autocatalytic) process, all the ants will rapidly choose the shorter path (Fig.12 D).

Referring to more recent work,[40] an interesting view distributed biological system ACS is presented. Therein, the ant Temnothorax albipennis uses a learning paradigm (technique) known as tandem running to lead another ant from the nest to food with signals between the two ants controlling both the speed and course of the run. That learning paradigm involves bidirectional feedback between teacher and pupil and considered as supervised learning, [24]. Interestingly, adopted animal learning principles herein, are recently applied for evaluation of some human educational issues[9][10].

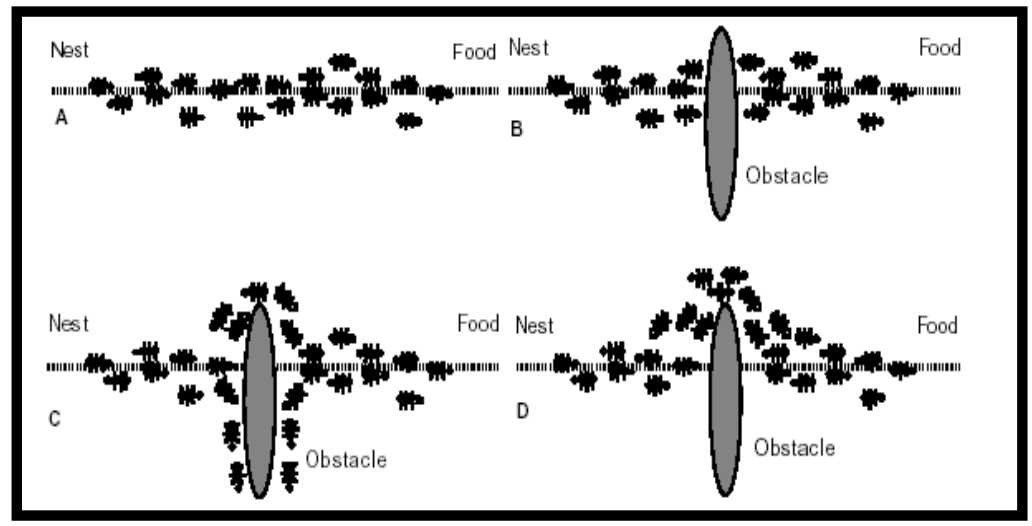

Fig.12:.Illustrates the process of transportation of food (from food source) to food store (nest).

Cooperative learning by Ant Colony System for solving TSP referring to Fig.12 which adapted from [42] , the difference between communication levels among agents (Ants) develops different outputs average speed to optimum solution. The changes of communication level are analogues to different values of $\lambda$ in odd sigmoid function as shown at equation (19) in below.. When the number of training cycles increases virtually to an [infinite value, the number of salivation drops obviously reach a saturation value additionally the pairing stimulus develops the learning process turned in accordance with Hebbian learning rule[22]. However in case of different values of $\lambda$ other than zero implicitly means that output signal is developed by neuron motors. Furthermore, by increasing of number of neurons which analogous to number of ant agents results in better learning performance for reaching accurate solution as graphically illustrated for fixed $\lambda[43][44]$.

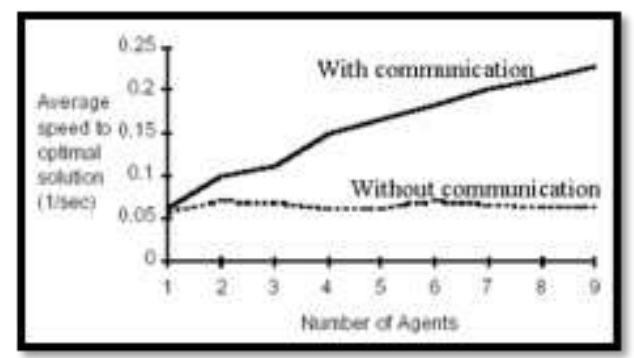

Fig. 13 :Illustrates performance of ACS with and without communication between ants \{adapted from[41] ) 
This different response speed to reach solution is analogous to different communication levels among agents (artificial ants) as shown at the Fig.10. It is worthy to note that communication among agents of artificial ants model develops different speed values to obtain an optimum solution of TSP, considering variable number of agents (ants).

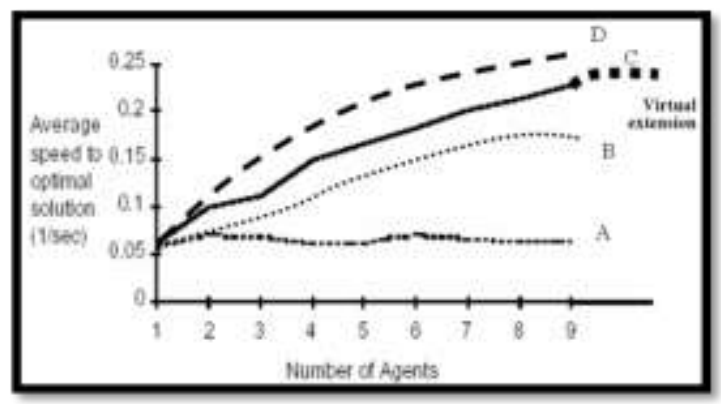

Fig.14: Communication determines a synergistic effect with different communication levels among agents leads to different values of average speed.

Consequently as this set of curves reaches different normalized optimum speed to get TSP solution (either virtually or actually) the solution is obtained by different number of ants, so this set could be mathematically formulated by following formula:

$$
f(n)=\alpha\left(\frac{1-e^{-\lambda n}}{1+e^{-\lambda n}}\right)
$$

Where $\alpha \ldots \ldots$. is an amplification factors representing asymptotic value for maximum average speed to get optimized solutions and $\lambda$ in the gain factor changing in accordance with communication between ants. Referring to the figure -- in below, the relation between number of neurons and the obtained achievement is given considering three different gain factor values $(0.5,1$, and 2$)$.

Referring to Fig.15, it illustrates obtained neural modeling results which declares an interesting qualitative comparative analogy between performance evaluation of behavioral ANN $\mathrm{s}$ modeling; versus smart optimization performance of Ant Colony System as presented at Figures (13\&14).More precisely, the gain factor values given at Fig.15 are analogous with the intercommunication level values inside the ACS given at Fig.13, and Fig.14.

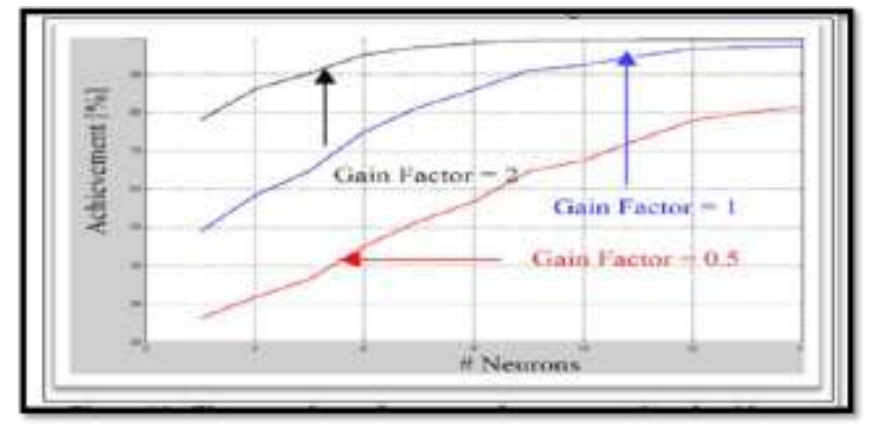

Fig. 15:. Illustrate students' learning achievement for different gain factors and intrinsically various number of neurons which measured for constant learning rate value $(\eta)=0.3$.

However by this mathematical formulation of that model normalized behavior it is shown that by changing of communication levels (represented by $\lambda$ ) that causes changing of the speeds for reaching optimum solutions. In given Fig. 16. in blow, it is illustrated that normalized model behavior according to following equation.

$$
\mathrm{y}(\mathrm{n})=(1-\exp (-\lambda \mathrm{i}(\mathrm{n}-1))) /(1+\exp (-\lambda \mathrm{i}(\mathrm{n}-1)))
$$

where $\lambda i$ represents one of gain factors (slopes) for sigmoid function. 


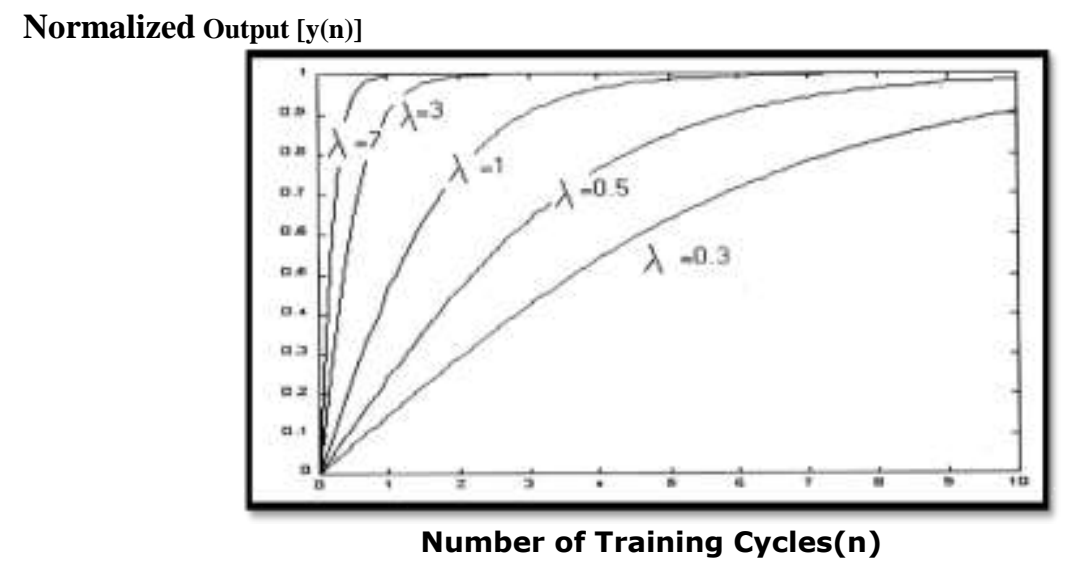

Fig. 16: Graphical representation of learning performance of model with different gain factor values $(\lambda)$

\section{Conclusions \& Future Work}

Herein, some conclusive remarks related to the obtained results are presented as well as some expected relevant future research directions that is carried out considering the effect of internal (intrinsic) learners' brain state as well as external environmental factors upon convergence of learning / training processes.

\subsection{Conclusions}

Through above presented performance evaluation approach, three interesting points are concluded subsequently to enhance quality of e-learning systems as follows:

- Evaluation of any e-learning system's quality following previously suggested measurement of learning convergence/response time. The experimental measured average of response time values (quantified evaluation), provides educationalists with a fair and unbiased judgment for any e-learning system (considering a pre-assigned achievement level).

- As consequence of above remark, relative quality comparison between two e-learning systems (on the bases of suggested metric measuring) is contributed by quantified performance evaluation.

- Modification of learning systems performance obtained by increment of learning rate value, which is expressed by the ratio between achievement level (testing mark) and the response learning time. This implies that learning rate could be considered as a modifying parameter contributes to both learning parameters (learning achievement level and learning convergence time response).

\subsection{Future Research Work}

The following are some research work directions that may be adopted in the future :

- Application of improved synaptic connectivity with random weight values in order to perform medically promising treatment of mentally disable learners.

- Simulation and modeling of complex educational issues such as deterioration of achievement levels at different learning systems due to non well prepared tutor .

- Study of ordering of teaching curriculum simulated as input data vector to neural systems. That improved both of learning and memory for the introduced simulated ANN model.

- Experimental measurement of learning systems' performance in addition to analytical modeling and simulation of these systems aiming to improve their quality.

Finally, more elaborate evaluation and assessment of individual differences phenomena that is needed critically for educational process.

\section{References}

[1] White House OSTP Issues Decade of the Brain Report, Maximizing Human Potential: 1990-2000.

[2] Grossberg, S. (ED.)" Neural Networks and Natural Intelligence" The MIT press, 1988, pp 1-5. 
[3] H.M. Hassan, Ayoub Al-Hamadi, and F. Al-Mohaya "On Quantifying Learning Creativity Using Artificial Neural Networks (A Nero-physiological Cognitive Approach)" Published at National Conference on Applied Cognitive Psychology held in India , Calcutta , 29 -30 November, 2007.

[4] H.M. Hassan, et al "On Enhancement of Reading Brain Performance Using Artificial Neural Networks' Modeling" published at Proceedings of the 2015 International Workshop on Pattern Recognition (ICOPR 2015) that held on May 4-5, 2015. Dubai, UAE.

[5] Ghonaimy M. A:, Al- Bassiouni, A. M. and Hassan, H. M.: learning ability in neural network model, Second International Conference on Artificial Intelligence Applications, Cairo, Egypt, 400- 413, Jan (1994), pp.22- 24

[6] Ghonaimy M. A., Al- Bassiouni, A. M. and Hassan, H. M.: learning of Neural Networks using Noisy Data, Second International Conference on Artificial Intelligence Applications, Cairo, Egypt, 400- 413, Jan (1994), pp.22-

[7] H.M. Hassan,"On Analysis of Quantifying Learning Creativity Phenomenon Considering Brain Synaptic Plasticity", published at WSSEC08 conference to be held on 18-22 August 2008, Derry, Northern Ireland.

[8] Swaminathan,N 2007 " Cognitive Ability Mostly Developed Before Adolescence, NIH Study Says. NIH announces preliminary findings from an effort to create a database that charts healthy brain growth and behavior "Scientific American letter, May 18, 2007.

[9] Hassan "On Analysis and Evaluation of Non-Properly Prepared Teachers Based on Character Optical Recognition Considering Neural Networks Modeling" Published at Proceedings of the International Conference on Pattern Recognition and Image Processing (ICPRIP'15) that held on March 16-17, 2015 Abu Dhabi (UAE).

[10] H.M. Hassan, and Ayoub Al-Hamadi "An Overview on Classrooms' Academic Performance Considering: Nonproperly Prepared Instructors, Noisy Learning Environment, and Overcrowded Classes (Neural Networks' Approach)" has been accepted for oral presentation and publication at the 6th International Conference on Distance Learning and Education (ICDLE 2015) in Pairs. Furthermore, this paper has been accepted to be published at the International Journal of Learning and Teaching in Vol.2, No.2, 2016 of IJLT.

[11] H.M. Hassan, and Ayoub Al-Hamadi, "On Comparative Analogy of Academic Performance Quality Regarding Noisy Learning Environment versus Non-properly Prepared Teachers Using Neural Networks' Modeling" published at the 7th International Conference on Education Technology and Computer (ICETC 2015) held on August 13-14, 2015, Berlin. Additionally, it has been published at the International Journal of Information and Education Technology in Vol.6, No.12. December 2016 of IJIET.

[12] Tsien, Joe. Z., "Building a brainier Mouse" Scientific American, Majallat Aloloom, vol. 17, No. 5, pp. 28- 35, (2001).

[13] Tsien, Joe. Z., Enhancing the link between Hebb`s coincidence detection and memory formation" in current opinion in Neurobiology, vol. 10, No. 2, (2000).

[14] Hassan, M.. H., "On Quantitative Mathematical Evaluation of Long Term Potentation and Depression Phenomena Using Neural Network Modeling” published at SIMMOD, 17- 19 Jan. 2005, pp. 237- 24.1

[15] Kandel, E: R. "Small Systems of Neuron“, Scientific American, Sept. 1979, vol.224, pp. 67-79.

[16] Douglas Fields, M. R. "Making Memories Sticks ",Majallat Aloloom, vol. 21 No. ${ }^{3}{ }_{4}$ pp. 18-25 (2005)

[17] H.M. Mustafa, et al. "On Assessment of Brain Function Adaptability in Open Learning Systems Using Neural Network Modeling (Cognitive Styles Approach), Published at The IEEE International Conference on Communications and Information Technology ICCIT-2011, held on Mar 29, 2011 - Mar 31, 2011, Aqaba, Jordan. pp. 229-237.

[18] Hassan "On optimal analysis and evaluation of time response in e-learning systems (neural networks approach)".Published at EDULEARN13, the 5th annual International Conference on Education and New Learning Technologies which will be held in Barcelona (Spain), on the 1st, 2nd and 3rd of July, 2013.

[19] H.M. Hassan, et al "Dynamical Evaluation Of Academic Performance In E-learning Systems Using Neural Networks Modeling (Time Response Approach)". published at IEEE EDUC[16]ON - Engineering Education 2014 held in 
Istanbul (Turkey), on the $3-5$ of April, 2014.Available on line at: http://ieeexplore.ieee.org/xpl/abstractAuthors.jsp?arnumber=6826150

[20] H.M. Hassan "On Brain Based Modeling of Blended Learning Performance Regarding learners' Self-assessment Scores Using Neural Networks (Brain Based Approach)" published at I A B L 20 1 6 : the IABL conference International Association for Blended Learning (IABL) will be held in Kavala Greece on 22-24 April 2016.

[21] H.M. Hassan, Mohammed H. Kortam, Ibrahim H. Assaf, Ayoub Al-Hamadi, Nada M. Al-Shenawy:"Optimal Estimation of Penalty Value for on Line Multiple Choice Questions Using Simulation of Neural Networks and Virtual Students' Testing". Published at the proceeding of UKSim-AMSS $16^{\text {th }}$ International Conference on Modeling and Simulation held at Cambridge University (Emmanuel College),on 26-28 March 2014.

[22] D.O. "The organization of Behavior", A Neuropsychological Theory, New York, Wiley, 1949.

[23] Al-Ajroush, “Associate Manual for Learners' Support in Face to Face Tuition" Arab Open University (KSA), May 2004..

[24] Haykin, S. Neural Networks, Englewood Cliffs, NJ: Prentice-Hall 1999.

[25] Fukaya, M., et. al. Two level Neural Networks: Learning by Interaction with Environment, 1 st ICNN; San Diego, 1988.

[26] H.M. Hassan "On Principles of Biological Information Processing Concerned with Learning Convergence Mechanism in Neural and Non-Neural Bio-Systems." Published at IEEE conference, CIMCA 2005 Vienna, Austria (28-30 Nov.2005).

[27] Ursula Dicke and Gerard Roth "Animal Intelligence and the Evolution of the Human Mind" Scientific American, Aug. 2008,in Mind \& Brain.

[28] Hassan "Building up bridges for natural inspired computational models across behavioral brain functional phenomena; and open learning systems" A tutorial presented at the International Conference on Digital Information and Communication Technology and its Applications (DICTAP2011) which held from June 21-23, 2011, at Universite de Bourgogne, Dijon, France.

[29] Hassan "Natural Inspired Computational Models for Open Learning" Published at the $5^{\text {th }}$ GUIDE INTERNATIONAL CONFERENCE held in Rome (Italy) 18 - 19 November 2011. Available online at: http://www.guideassociation.org/proceedings/Guide_2011/

[30] H.M.Hassan, et al. "On Comparison Between Swarm Intelligence Optimization and Behavioral Learning Concepts Using Artificial Neural Networks (An over view)", published at the 12th World Multi-Conference on Systemics, Cybernetics and Informatics: WMSCI 2008 The 14th International Conference on Information Systems Analysis and Synthesis: ISAS 2008 June $29^{\text {th }}$ - July $2^{\text {nd }}, 2008$ - Orlando, Florida, USA.

[31] H.M. Hassan, "Comparative Performance Analysis for Selected Behavioral Learning Systems versus Ant Colony System Performance (Neural Network Approach)". Published at the International Conference on Machine Intelligence ICMI 2015.Held on Jan 26-27, 2015, in Jeddah, Saudi Arabia.

[32] H.M. Hassan," A Comparative Analogy of Quantified Learning Creativity in Humans Versus Behavioral Learning Performance in Animals: Cats, Dogs, Ants, and Rats.(A Conceptual Overview), to be published at WSSEC08 conference to be held on 18-22 August 2008, Derry, Northern Ireland.

[33] H.M. Hassan, et al "Comparative Performance Analysis and Evaluation for One Selected Behavioral Learning System versus an Ant Colony Optimization System" Published at the Proceedings of the Second International Conference on Electrical, Electronics, Computer Engineering and their Applications (EECEA2015), Manila, Philippines, on Feb. 12$14,2015$.

[34] Chhabra A,Singh "Analysis \& Integrated Modeling of the Performance evaluation Technique for Evolutionary Parallel Systems ",Published at International Journal of Computer Science and Security IJCSS Volume .(1):Issue(1),2008.

[35] Caudill, M. "Neural Networks primer, part 1” AI Expert, Aug. 1989, pp 1-7 
[36] Kohonen, T. Physiological interpretation of the self organizing map algorithm, Neural Networks 6, pp. 895 - 909 (1993) https://doi.org/10.1016/s0893-6080(09)80001-4

[37] Kohonen, T. "overture, Self- organizing Neural Networks, recent advances and applications, Physica- Verlag Heidelberg, New York, U. Seifert an Jain, L. C. (Eds.) (2002).

[38] Kohonen, T. self organizing maps, $3^{\text {rd }}$ ed. Springer, London, 2001.

[39] Chhabra A,Singh "Analysis \& Integrated Modeling of the Performance evaluation Technique for Evolutionary Parallel Systems ",Published at International Journal of Computer Science and Security IJCSS Volume .(1):Issue(1),2008.

[40] Caudill, M. "Neural Networks primer, part 1" AI Expert, Aug. 1989, pp 1-7

[41] Hassan,2012]"On performance evaluation of brain based learning processes using neural networks," published at 2012 IEEE Symposium on Computers and Communications (ISCC), pp. 000672-000679, 2012 IEEE Symposium on Computers and Communications (ISCC), 2012. Available online at : http://www.computer.org/csdl/proceedings/iscc/2012/2712/00/IS264-abs.html

[42] Dorigo, M. et al ,1997: Ant colonies for the traveling sales man problem at web site www.iridia.ulb.ac.be/dorigo/aco/aco.html

[43] Rechardson T.,Franks N.R. "Teaching in tandem-running ants", Nature, Jan 2006,12;439(7073):153.

[44] Eric Bonabeau, and Guy Theraulaz, "Swarm Smarts" Majallat Aloloom, May 2001, vol 17, No 5, pp 4-12.sssss 